\title{
Adaptação transcultural para o Português do questionário de autorrelato da função sensorial e motora de S4-5 (S4-5Q) em pessoas com lesão medular espinhal
}

Cross-cultural Adaptation of Self-report S4-5 Sensory and Motor Function Questionnaire (S4-5Q) in People with Spinal Cord Injury to Portuguese

\author{
Francielle Romanini1일 Thays de Souza Lima ${ }^{20}$ \\ ${ }^{1}$ Núcleo de Pesquisa em Lesão Medular, Universidade do Estado de \\ Santa Catarina, Programa de Pós-Graduação em Neurociência, \\ Universidade Federal de Santa Catarina, Florianópolis, SC, Brasil \\ ${ }^{2}$ Núcleo de Pesquisa em Lesão Medular, Universidade do Estado de \\ Santa Catarina, Florianópolis, SC, Brasil \\ ${ }^{3}$ Departamento de Cinesiologia e Saúde Comunitária, Faculdade de \\ Ciências Aplicadas à Saúde, Universidade de Illinois em Urbana- \\ Champaign, Urbana, Illinois, Estados Unidos
}

\author{
Libak Abou ${ }^{3(1)}$ Jocemar Ilha1일
}

Rev Bras Ortop 2022;57(3):384-391.

\begin{abstract}
Resumo
Objetivo O "Self-report S4-5 Sensory and Motor Function Questionnaire (S4-5Q)" é um breve questionário de avaliação da função dos segmentos sacrais S4 e S5 após uma lesão medular (LM), cuja finalidade é substituir o exame de toque retal. O objetivo do presente estudo foi realizar uma adaptação transcultural do questionário S4-5Q para a língua portuguesa do Brasil e investigar a confiabilidade teste-reteste desta versão. Método A tradução e a adaptação transcultural foram realizadas utilizando a metodologia de tradução e retrotradução. Inicialmente, as traduções foram realizadas por três tradutores independentes. Sua síntese foi então submetida a um comitê de especialistas (profissionais

Palavras-chave

- lesão da medula espinhal

- região sacral

- comparação transcultural

- pesquisas e questionários de saúde com experiência em LM). Em seguida, a retrotradução para o idioma original foi enviada ao autor original, sendo que a versão final foi concluída após todas as adaptações. Para a confiabilidade teste-reteste, foram recrutados 24 indivíduos com lesão medular espinal (LME) em fase crônica (2 avaliações com intervalo de 7 a 14 dias entre elas). A análise estatística foi realizada no IBM-SPSS (Version 20, SPSS Inc, Chicago, IL, USA) com dados prétabulados no programa Microsoft Excel (Microsoft Corporation, Redmond, WA, EUA). A confiabilidade foi testada por meio do coeficiente Kappa de Cohen, e a consistência interna foi medida através do $\alpha$ de Cronbach, ambas adotando $p<0,05$ como significante.
\end{abstract}

Trabalho desenvolvido no Departamento de Fisioterapia da Faculdade de Ciências da Saúde e do Esporte da Universidade do Estado de Santa Catarina (UDESC), Florianópolis, SC, Brasil. recebido

22 de Fevereiro de 2021

aceito

08 de Março de 2021

Publicado on-line

Setembro 20, 2021
DOI https://doi.org/

10.1055/s-0041-1735233.

ISSN 0102-3616.
(C) 2021. Sociedade Brasileira de Ortopedia e Traumatologia. All rights reserved.

This is an open access article published by Thieme under the terms of the Creative Commons Attribution-NonDerivative-NonCommercial-License, permitting copying and reproduction so long as the original work is given appropriate credit. Contents may not be used for commercial purposes, or adapted, remixed, transformed or built upon. (https://creativecommons.org/ licenses/by-nc-nd/4.0/)

Thieme Revinter Publicações Ltda., Rua do Matoso 170, Rio de Janeiro, RJ, CEP 20270-135, Brazil 


\begin{abstract}
Keywords

- spinal cord injury

- sacral region

- cross-cultural comparison

- surveys and questionnaires

Objective The "Self-report S4-5 sensory and motor function questionnaire (S4-5Q)" is a short questionnaire that aims to assess the function of the sacral segments, S4 and $\mathrm{S} 5$, after a spinal cord injury $(\mathrm{SCl})$, with the purpose of replacing the rectal exam test. The aim of the present study was to carry out a cross-cultural adaptation of the S4-5Q to the Brazilian Portuguese language and to investigate the test-retest reliability of this version.

Method The translation and cross-cultural adaptation was performed using the methodology of translation and backtranslation. Initially, translations were done by three independent translators. Their synthesis was then submitted to an expert committee for analysis ( $\mathrm{SCl}$ health professionals); then, the backtranslation to the original language was sent to the original author and, after all the adjustments, the final version was completed. For test-retest reliability, 24 individuals with chronic $\mathrm{SCl}$ were recruited ( 2 evaluations with an interval of 7 to 14 days between them). Statistical analysis was performed using IBM-SPSS (Version 20, SPSS Inc, Chicago, IL, USA) with data pretabulated in Microsoft Excel (Microsoft Corporation, Redmond, WA, USA). Reliability was tested with Cohen Kappa, and internal consistency with Cronbach $\alpha$, both adopting $p<0.05$ as significant.

Results In the reliability analysis, the results of all questions showed almost perfect agreement (Kappa $>0.81$ ) and good internal consistency (Cronbach $\alpha$ : 0.65-0.77) between the questions and final classification.

Conclusion The cross-culturally adapted S4-5Q is reliable to be applied to the Brazilian population with chronic $\mathrm{SCl}$ to determine their $\mathrm{S} 4-5$ sensory and motor function.
\end{abstract}

Resultados $\mathrm{Na}$ análise de confiabilidade, os resultados de todas as perguntas apresentaram concordância quase perfeita (Kappa $>0,81$ ) e boa consistência interna ( $\alpha$ de Cronbach: $0,65-0,77$ ) entre as perguntas e a classificação final.

Conclusão O questionário S4-5Q adaptado culturalmente é confiável, podendo ser aplicado à população brasileira com LME crônica, com o objetivo de avaliar a função sensorial e motora nos segmentos sacrais S4-S5.

\section{Introdução}

A American Spinal Injury Association (ASIA, na sigla em inglês) Impairment Scale (AIS, na sigla em inglês) é a medida clínica mais amplamente utilizada para avaliar e classificar a função neurológica de indivíduos com lesão medular espinhal (LME). ${ }^{1}$ Esta classificação é feita por meio dos Padrões Internacionais de Classificação Neurológica para Exame de Lesão da Medula Espinhal (International Standards for Neurological Classification for Spinal Cord Injury [ISNCSCI, na sigla em inglês]). Esta classificação foi desenvolvida para avaliar especificamente $\mathrm{o}(\mathrm{s})$ segmento(s) afetado(s) da medula espinhal e a extensão da lesão. ${ }^{2}$ Entre outros aspectos, o ISNCSCI pode diferenciar clinicamente entre uma lesão completa e incompleta a partir das funções sensoriais e motoras remanescentes nos segmentos sacrais $\mathrm{S} 4-\mathrm{S} 5 .^{2-5}$

Com o objetivo de avaliar e determinar o grau de comprometimento motor e sensorial dos segmentos sacrais S4-S5, deve ser realizada uma avaliação por meio do exame de toque retal, o qual é essencial para avaliar esta região por meio do ISNCSCI. ${ }^{2,6}$ No entanto, na prática clínica, existem algumas barreiras para a realização do exame físico dos segmentos S4-
S5. Entre estas limitações, as mais comuns são a necessidade de um profissional treinado, de um local adequado para o teste e de materiais adicionais. Além disso, trata-se de um exame invasivo, com riscos associados a sangramento retal e estimulação das evacuações, podendo ocorrer uma evacuação durante o teste. ${ }^{4}$ Considerando que estas barreiras de aplicação do exame podem inviabilizar a aplicação integral deste, foram propostos métodos alternativos de classificação dos segmentos sacrais ${ }^{7-10}$; entre eles, o questionário de autorrelato da função sensorial e motora dos segmentos sacrais S4-S5 (S4$5 \mathrm{Q})$, que provou ser uma alternativa viável. ${ }^{4}$

O questionário S4-5Q é composto por quatro questões, sendo três relacionadas às funções sensoriais e uma à função motora. ${ }^{4}$ Este questionário foi desenvolvido em inglês e mostrou-se acurado para avaliar a função motora e sensorial dos segmentos S4-S5 em indivíduos com $>12$ meses de lesão (na fase pós-lesão crônica). ${ }^{4}$ Porém, para aplicar este questionário à população brasileira, é necessário adaptá-lo transculturalmente, desde a adaptação da linguagem do ponto de vista cultural e conceitual, visando trazer o mais próximo possível da realidade da população de interesse. ${ }^{11}$ Portanto, o objetivo do presente estudo foi 


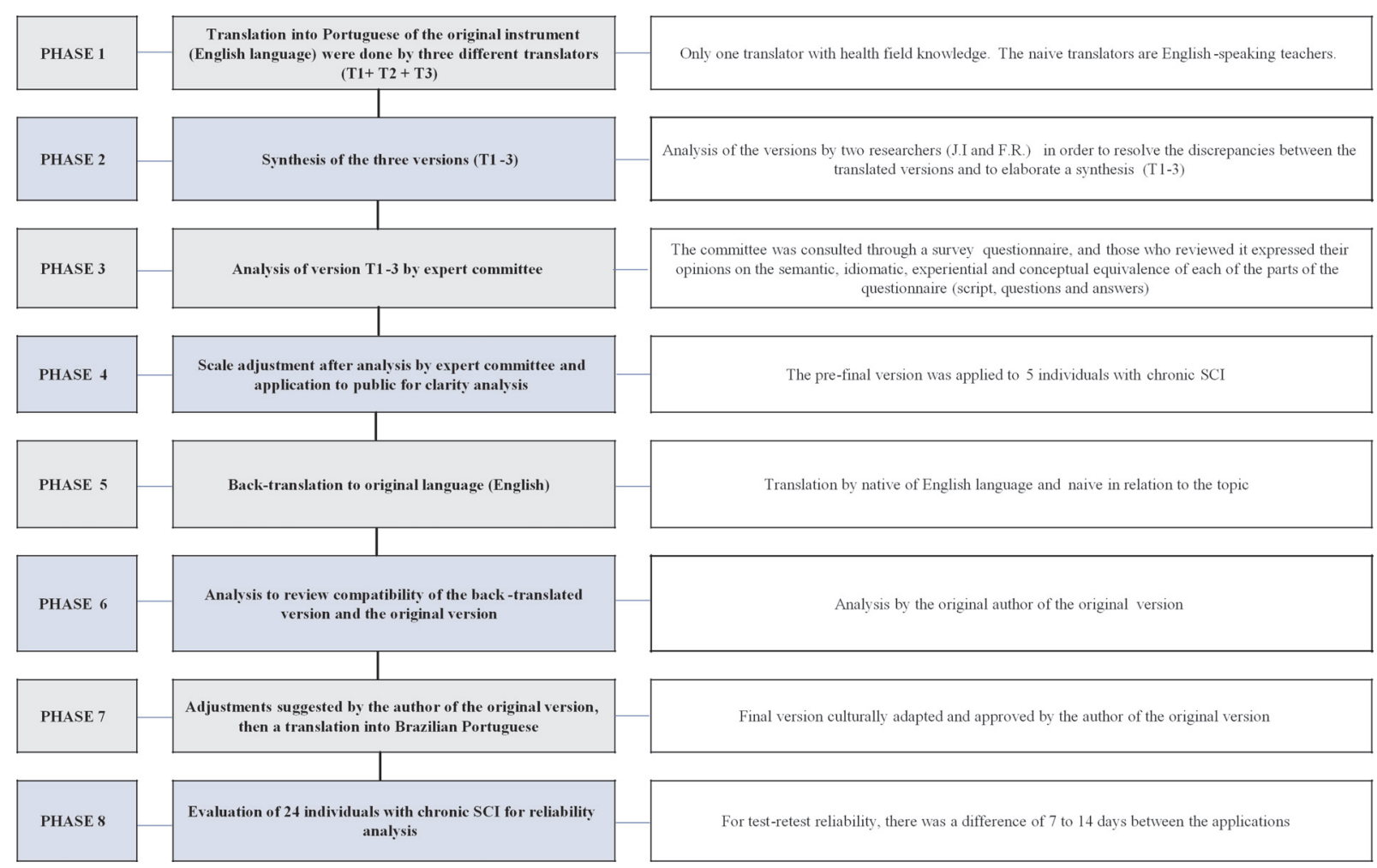

Fig. 1 Etapas da tradução, adaptação cultural e análise da confiabilidade do questionário (S4-5Q) de autorrelato para o português, para avaliar a função sensorial e motora dos segmentos sacrais S4-S5.

realizar uma adaptação transcultural do questionário S4-5Q para o idioma português brasileiro, assim como testar a confiabilidade teste-reteste da versão traduzida e adaptada.

\section{Métodos}

\section{Desenho do Estudo}

Trata-se de um estudo transversal aprovado pelo comitê local (CAAE 90139118.7.0000.0118). O presente estudo foi desenhado para realizar uma adaptação transcultural do questionário S4-5Q de acordo com a metodologia proposta por Beaton et al. ${ }^{11}$ Foi realizada uma série de etapas metodológicas sistematizadas, a qual inclui o procedimento de tradução linguística e adaptação transcultural, abrangendo várias fases (descritas na -Figura 1). Além disso, foi adotada neste estudo a taxonomia dos Padrões Baseados em Consenso para a Seleção de Instrumentos de Medição de Saúde (COSMIN). ${ }^{12}$

Após a adaptação transcultural, a versão final traduzida para o português brasileiro foi utilizada para análise da confiabilidade teste-reteste e consistência interna. Nesta etapa, o questionário foi aplicado a 24 indivíduos com LME, por meio de entrevista face a face. Os critérios de inclusão foram diagnóstico clínico de LME há $>1$ ano e pacientes $>18$ anos. Após um período de 7 a 14 dias, os indivíduos foram reavaliados com o questionário, porém 5 pessoas não compareceram no dia do reteste.

\section{Análise de Dados e Estatística}

Para determinar a concordância entre os especialistas, foi utilizado o percentual de respostas ao questionário, levando em consideração que existiam apenas duas opções de resposta ("Concordo" e "Não Concordo"). A consistência interna entre os itens da escala traduzida foi analisada por meio do coeficiente $\alpha$ de Cronbach, interpretado por valores entre zero e 1 , onde os valores foram considerados de boa consistência quando $\alpha \geq 0,70$. A confiabilidade teste-reteste foi analisada por meio do teste estatístico Kappa de Cohen das respostas, para cada questão. Valores com $p<0,05$ foram considerados significativos, com a seguinte interpretação: leve, de 0,01 a 0,20 ; regular, de 0,21 a 0,40; moderado, de 0,41 a 0,60 ; substancial, de 0,61 a 0,80 ; e quase perfeito de 0,81 a $1,0 .{ }^{13}$

\section{Resultados}

A primeira etapa consistiu na tradução e adaptação cultural do questionário S4-5Q para o idioma português brasileiro. Houve divergências entre os tradutores quanto aos termos utilizados, embora nenhuma dessas diferenças alterasse o significado original das frases. Dentre as adaptações necessárias para sintetizar as três versões, houve a necessidade de ajustar o tempo verbal entre as versões, a fim de que houvesse padronização em todas as questões. Além disso, a tradução da expressão "apertar" foi definida como "contrair" pela equipe de pesquisa, com o objetivo de deixar claro o entendimento da função muscular questionada.

No roteiro, os termos em português "em volta" foram substituídos por "ao redor", "queira por favor me informar" para "me informe por favor", e "um exame retal" para "um exame de toque retal", simplificando as expressões e, 
consequentemente, proporcionando uma melhor compreensão. O mesmo ocorreu na pergunta $1 \mathrm{~A}$, em que a palavra "levemente" foi substituída pelas palavras "tocasse levemente".

A substituição na questão $1 \mathrm{C}$ foi a expressão em português “fizesse pressão", adaptando-a para "aplicasse pressão". O verbo foi alterado para esclarecer o entendimento da versão em português. Na questão 2, foi alterado o termo "para realizar o enema", substituído por "o enema”, já que, assim, mantém a equivalência idiomática com a versão original.

\section{Revisão do Comitê de Especialistas}

Após os ajustes, a síntese foi escrita e submetida à análise do comitê de especialistas, onde 12 profissionais responderam a um questionário digital. Entre eles, havia 8 fisioterapeutas, 3 enfermeiras e 1 neurologista. Apenas $8 \%$ destes profissionais possuíam bacharelado/diploma de graduação; o restante declarou possuir residência na área (8\%), mestrado (50\%) ou doutorado (33\%). O tempo médio do exercício profissional foi de $\sim 9$ anos.

Quanto à análise do questionário, o roteiro teve $100 \%$ de concordância em todos os aspectos. A questão $1 \mathrm{~A}$ obteve concordância semântica de $91,7 \%$ e, nas demais equivalências, alcançou $100 \%$. A sugestão nesta pergunta foi a de que 0 termo utilizado ("você sentiria") poderia induzir a resposta e deveria ser revisto pela equipe técnica. A questão 1B apresentou concordância semântica de $83,3 \%$ e, para as demais, a equivalência foi de $91,7 \%$. A partir da discordância, sugeriu-se que o instrumento utilizado no teste poderia variar, sendo necessária a revisão do item descrito. A questão $1 \mathrm{C}$ apresentou concordância experiencial e conceitual total; porém, quanto à semântica e à idiomática, apresentou 91,7\%. As sugestões feitas foram em relação ao contexto da "pressão" exercida e à forma literal mais correta de ser compatível com a prática. A questão 2 mostrou 100\% de concordância em todas as equivalências.

Após os ajustes sugeridos pelo comitê de especialistas, foi formulada a versão pré-final, que não exigiu ajustes durante a aplicação do questionário com os cinco pacientes com LME, uma vez que eles conseguiram compreender e relataram compreensão das perguntas. A versão retrotraduzida foi julgada pelo autor original do questionário, que sugeriu incluir o termo "segurança" após o termo "alfinete" na questão 1B ("Você poderia distinguir entre as extremidades com ou sem ponta de um alfinete de segurança?"), assim como ajustar o termo "realizar" para "segurar" na questão 2 ("ou segurar um enema”). As versões da escala original (em inglês), a síntese T13 e a versão pré-final são mostradas na - Tabela 1. O formulário de registro da versão final em português do questionário S4-5Q é apresentado no Apêndice A.

\section{Análise de Confiabilidade}

A versão final foi utilizada para avaliar uma amostra de 24 pessoas portadoras de LME crônica (> 12 meses), selecionadas por conveniência para análise de confiabilidade testereteste. As características dos participantes estão descritas na - Tabela 2. Os participantes apresentaram tempo médio de lesão de $11,7 \pm 10,5$ anos e distribuição homogênea quanto ao gênero. Quanto ao nível e complexidade da lesão, houve predomínio das lesões incompletas (75\%) e torácicas (75\%). Dentre os indivíduos incluídos na amostra, 50\% foram classificados com AIS C, enquanto os demais foram classificados entre AIS A (29\%), AIS B (8\%) e AIS D (12,5\%).

Na análise estatística de confiabilidade, todas as perguntas apresentaram concordância quase perfeita (Kappa $>0,81$ ) (-Tabela 3). O percentual de concordância para a Pergunta 1A foi de $82 \%$, para 1B $91 \%$, e para 1 C e D $90 \%$, com $p<0,001$ para ambas. $O$ resultado da escala utilizada para avaliar entre lesão completa ou incompleta apresentou concordância substancial (78\%; $p<0,001)$. A análise de consistência interna detectou $\alpha$ de Cronbach de 0,65 entre os itens do questionário e $\alpha$ de Cronbach de 0,77 entre as perguntas e o resultado da classificação final. Quando analisada a probabilidade de respostas por pessoa, apenas três responderam de forma diferente no reteste, sendo que todas apresentavam AIS C.

\section{Discussão}

O instrumento de autorrelato S4-5Q desenvolvido por Harvey et al., ${ }^{4}$ inicialmente testado na população australiana com LME, é útil para examinar a região perianal e classificar a LME como completa ou incompleta, quando não for possível realizar o exame de toque retal. Assim, buscou-se trazer para a comunidade de língua portuguesa uma versão padronizada e adaptada transculturalmente deste questionário no idioma português brasileiro, já que se considera que a tradução literal poderia prejudicar a padronização dos resultados e a interpretação das evidências dos artigos. Assim, o objetivo do nosso estudo foi realizar a tradução e a adaptação do questionário para a língua portuguesa, viabilizando este método diagnóstico também para a população brasileira portadora de LME.

Para este processo, foi aplicada a metodologia descrita por Beaton et al. ${ }^{11}$ Este método possui consistência e detalhamento metodológico, promovendo uma melhor adaptação transcultural durante o processo de tradução, sem distorcer o construto da escala original. No entanto, a ordem dos processos de análise do comitê de especialistas e da retrotradução foi alterada pelos autores. Isto se deve ao fato de que as alterações feitas melhorariam a interação com o autor da versão original, já que todas as análises da língua portuguesa foram feitas na fase de tradução primária. No presente estudo, somente após a extração de todas as análises aplicáveis da versão pré-final é que se procedeu ao processo de retrotradução e, consequentemente, à análise pelo autor da versão original. Vale ressaltar que, mesmo tendo feito ajustes para a versão em português, o processo de adaptação transcultural adotado no nosso estudo manteve a equivalência entre a versão brasileira e a versão original do questionário, já que a comparação do questionário original e a retrotradução foi realizada pelo autor da versão original.

A consistência interna entre os itens do instrumento apresentou um $\alpha$ de Cronbach de 0,65 , ou seja, $<0,70$. Isto pode ter ocorrido devido às diferenças no construto interno do questionário, uma vez que ele possui questões de motricidade e sensibilidade. Porém, é possível considerar uma boa consistência interna do questionário, pois ele apresentou valores $\alpha$ de 
Tabela 1 Apresentação das alterações da versão original à síntese das traduções e da versão pré-final, após os ajustes propostos na avaliação das equivalências semânticas, idiomáticas, culturais e conceituais para os itens do questionário S4-5Q

\begin{tabular}{|c|c|c|}
\hline Documento original em Inglês & Síntese da tradução para o Português & $\begin{array}{l}\text { Versão pré-final após ajustes propostos } \\
\text { por profissionais de reabilitação }\end{array}$ \\
\hline $\begin{array}{l}\text { Script for administration of the S4-5 sen- } \\
\text { sory and motor questionnaire (S45Q): } \\
\text { I am going to ask you } 4 \text { questions about } \\
\text { your sensation and strength in and } \\
\text { around anus. } \\
\text { The questions are of a personal nature. } \\
\text { If at any time you feed uncomfortable } \\
\text { answering any questions, or for any rea- } \\
\text { son would prefer not to answer these } \\
\text { questions, please let me know and I will } \\
\text { cease asking anything more. } \\
\text { The questions are important for deter- } \\
\text { mining the extent of your spinal cord } \\
\text { injury. } \\
\text { They tell us whether nerve messages are } \\
\text { getting past your injury all the way to the } \\
\text { end of your spinal cord. } \\
\text { The only other way to gain the same } \\
\text { information is for a clinician to test the } \\
\text { strength and sensation of your anus. This } \\
\text { involves a rectal examination. } \\
\text { The questions will help avoid the need for } \\
\text { a rectal examination although regardless, } \\
\text { the final decision about a rectal exami- } \\
\text { nation is always yours. } \\
\text { Sometimes irrespective of your answers } \\
\text { to the questions, we recommend that you } \\
\text { have a rectal examination. } \\
\text { If you do not understand any of the } \\
\text { terminology we are using, then please } \\
\text { just answer 'uncertain'. } \\
\text { Are you happy to proceed and for me to } \\
\text { ask you the questions? }\end{array}$ & $\begin{array}{l}\text { Roteiro para administração do questio- } \\
\text { nário sensitivo e motor S4-5 (S45Q): } \\
\text { Eu vou Ihe fazer } 4 \text { perguntas sobre a sua } \\
\text { sensibilidade e força dentro e em volta a } \\
\text { do ânus. As perguntas são de natureza } \\
\text { pessoal. } \\
\text { Se em algum momento você se sentir } \\
\text { constrangido ao responder a qualquer } \\
\text { pergunta, ou, por qualquer razão, prefira } \\
\text { não respondê-las, queira por favor me } \\
\text { informar, }{ }^{\text {a }} \text { que eu interromperei o ques- } \\
\text { tionamento. } \\
\text { As perguntas são muito importantes para } \\
\text { determinar a extensão da sua lesão da } \\
\text { medula espinhal. Elas nos informam se as } \\
\text { mensagens nervosas estão conseguindo } \\
\text { passar pela sua lesão até o final da sua } \\
\text { medula espinhal. } \\
\text { A outra maneira de se obter a mesma } \\
\text { informação é através de um clínico tes- } \\
\text { tando a sensibilidade e força de seu ânus. } \\
\text { Isso envolve um exame retal. } \\
\text { As perguntas nos ajudam a evitar a } \\
\text { necessidade de um exame retal, muito } \\
\text { embora a decisão final sobre este tipo de } \\
\text { exame seja sempre sua. } \\
\text { Eventualmente, independentemente de } \\
\text { suas respostas às perguntas, nós reco- } \\
\text { mendamos que você se submeta a um } \\
\text { exame retal. Caso você não compreenda } \\
\text { a terminologia que estamos utilizando, } \\
\text { queira por favor responder "não tenho } \\
\text { certeza”. } \\
\text { Você gostaria de prosseguir e sente-se } \\
\text { confortável com as perguntas? }\end{array}$ & $\begin{array}{l}\text { Roteiro para administração do questio- } \\
\text { nário sensitivo e motor S4-5 (S45Q): } \\
\text { Eu vou Ihe fazer } 4 \text { perguntas sobre a sua } \\
\text { sensibilidade e força dentro e ao redor do } \\
\text { ânus. As perguntas são de natureza pes- } \\
\text { soal. Se em algum momento você se } \\
\text { sentir constrangido ao responder a qual- } \\
\text { quer pergunta, ou, por qualquer razão, } \\
\text { prefira não respondê-las, me informe por } \\
\text { favor, a que eu interromperei o questio- } \\
\text { namento. } \\
\text { As perguntas são muito importantes para } \\
\text { determinar a extensão da sua lesão da } \\
\text { medula espinhal. Elas nos informam se as } \\
\text { mensagens nervosas estão conseguindo } \\
\text { passar pela sua lesão até o final da sua } \\
\text { medula espinhal. } \\
\text { A outra maneira de se obter a mesma } \\
\text { informação é através de um clínico tes- } \\
\text { tando a sensibilidade e força de seu ânus. } \\
\text { Isso envolve um exame de toque retal. } \\
\text { As perguntas nos ajudam a evitar a } \\
\text { necessidade de um exame retal, muito } \\
\text { embora a decisão final sobre este tipo de } \\
\text { exame seja sempre sua. Eventualmente, } \\
\text { independentemente de suas respostas às } \\
\text { perguntas, nós recomendamos que você } \\
\text { se submeta a um exame retal. Caso você } \\
\text { não compreenda a terminologia que } \\
\text { estamos utilizando, queira por favor re- } \\
\text { sponder "não tenho certeza". } \\
\text { Você gostaria de prosseguir e sente-se } \\
\text { confortável com as perguntas? }\end{array}$ \\
\hline $\begin{array}{l}\text { Question 1a: Could you feel anything if I } \\
\text { were to lightly touch the skin just around } \\
\text { your anus with cotton wool? }\end{array}$ & $\begin{array}{l}\text { Pergunta 1a: Você sentiria alguma coisa } \\
\text { se eu tocasse com algodão, levemente, }{ }^{a} \text { a } \\
\text { pele bem ao redor do seu ânus? }\end{array}$ & $\begin{array}{l}\text { Pergunta 1a: Você sentiria alguma coisa } \\
\text { se eu tocasse levemente }{ }^{a} \text { com algodão a } \\
\text { pele bem }{ }^{b} \text { ao redor do seu ânus? }\end{array}$ \\
\hline $\begin{array}{l}\text { Question 1b: Could you distinguish be- } \\
\text { tween the sharp and blunt end of safety } \\
\text { pin if I were alternatively touch you with } \\
\text { the sharp and blunt end of a safety pin on } \\
\text { the skin just around your anus? }\end{array}$ & $\begin{array}{l}\text { Pergunta 1b: Você poderia distinguir } \\
\text { entre as extremidades com ou sem ponta } \\
\text { de um alfinete, se a pele ao redor de seu } \\
\text { ânus fosse tocada alternadamente por } \\
\text { elas? }\end{array}$ & $\begin{array}{l}\text { Pergunta } 1 \text { b: Você poderia distinguir } \\
\text { entre as extremidades com ou sem ponta } \\
\text { de um alfinete, se a pele ao redor de seu } \\
\text { ânus fosse tocada alternadamente por } \\
\text { elas? }\end{array}$ \\
\hline $\begin{array}{l}\text { Question 1c: Could you feel pressure if I } \\
\text { were to insert a gloved finger into your } \\
\text { rectum and apply pressure to the wall of } \\
\text { your anus? }\end{array}$ & $\begin{array}{l}\text { Pergunta 1c: Você sentiria pressão se eu } \\
\text { inserisse um dedo com luvas no seu reto e } \\
\text { fizesse pressão }^{\text {a }} \text { na parede do seu ânus? }\end{array}$ & $\begin{array}{l}\text { Pergunta 1c: Você sentiria pressão se eu } \\
\text { inserisse um dedo com luvas no seu reto e } \\
\text { aplicasse pressão }^{\text {a }} \text { na parede do seu ânus? }\end{array}$ \\
\hline $\begin{array}{l}\text { Question 2: Can you tighten the muscles } \\
\text { of your anus as if you were going to hold } \\
\text { in a toilet motion or enema, or prevent } \\
\text { the passing of wind? }\end{array}$ & $\begin{array}{l}\text { Pergunta 2: Você consegue contrair os } \\
\text { músculos do seu ânus como se fosse } \\
\text { segurar a vontade de evacuar ou para } \\
\text { realizar o enema, }^{\text {a }} \text { ou para evitar a saída }\end{array}$ & $\begin{array}{l}\text { Pergunta 2: Você consegue contrair os } \\
\text { músculos do seu ânus como se fosse } \\
\text { segurar a vontade de evacuar ou o ene- } \\
\text { ma, ou para evitar a saída de gazes? }\end{array}$ \\
\hline Yes & Sim & Sim \\
\hline No & Não & Não \\
\hline Uncertain & Não tenho certeza & Não tenho certeza \\
\hline (circle appropriate answer) & (circule a resposta apropriada) & (circule a resposta apropriada) \\
\hline
\end{tabular}

Legenda: (a) As palavras que sofreram ajustes propostos pelo autor estão sublinhadas, enquanto (b) as palavras em negrito foram alteradas após análise dos especialistas. 
Tabela 2 Caracterização da amostra dos indivíduos com lesão medular em que foi aplicada a versão final da escala adaptada transculturalmente para o idioma português

\begin{tabular}{|l|l|l|l|l|l|}
\hline Participantes $(\mathbf{n}=\mathbf{2 4})$ & Tempo desde a lesão (anos) & Gênero & Classificação & Nível de lesão & AIS \\
\hline 01 & 1 & M & Incompleta & Torácica alta & C \\
\hline 02 & 9 & F & Incompleta & Torácica alta & A \\
\hline 03 & 2 & M & Completa & Torácica baixa & A \\
\hline 04 & 3 & M & Incompleta & Torácica alta & D \\
\hline 05 & 8 & M & Completa & Torácica alta & D \\
\hline 06 & 3 & F & Incompleta & Torácica baixa & C \\
\hline 07 & 12 & M & Incompleta & Cervical & B \\
\hline 08 & 12 & F & Incompleta & Torácica alta & A \\
\hline 09 & 3 & F & Completa & Torácica alta & A \\
\hline 10 & 15 & F & Incompleta & Torácica baixa & C \\
\hline 11 & 33 & F & Incompleta & Torácica alta & C \\
\hline 12 & 11 & M & Incompleta & Torácica baixa & C \\
\hline 13 & 5 & M & Incompleta & Torácica baixa & B \\
\hline 14 & 10 & M & Completa & Torácica baixa & A \\
\hline 15 & 21 & F & Incompleta & Cervical & C \\
\hline 16 & 2 & F & Incompleta & Torácica baixa & C \\
\hline 17 & 38 & M & Incompleta & Cervical & C \\
\hline 18 & 33 & M & Incompleta & Cervical & D \\
\hline 19 & 23 & M & Completa & Torácica alta & A \\
\hline 20 & 10 & F & Incompleta & Torácica baixa & C \\
\hline 21 & 10 & M & Incompleta & Lombar & C \\
\hline 22 & 7 & F & Incompleta & Lombar & C \\
\hline 23 & 6 & F & Completa & Torácica baixa & A \\
\hline 24 & 3 & Incompleta & Torácica alta & C \\
\hline
\end{tabular}

Abreviações: AIS, American Spinal Injury Association impairment scale; F, feminino; M, masculino.

Tabela 3 Análise estatística da confiabilidade teste-reteste

\begin{tabular}{|l|l|l|}
\hline \multirow{2}{*}{ Pergunta } & Teste-reteste \\
\cline { 2 - 3 } & Concordância (Kappa) & valor-p \\
\hline 1A & 0,82 & $p<0,001$ \\
\hline 1B & 0,91 & $p<0,001$ \\
\hline $1 C$ & 0,90 & $p<0,001$ \\
\hline 2 & 0,90 & $p<0,001$ \\
\hline Classificação & 0,78 & $p<0,001$ \\
\hline
\end{tabular}

Valor de $p<0,05$ foi considerado significativo.

A classificação entre lesão completa e incompleta das outras perguntas também foi testada.

Cronbach $>0,70$ para a análise entre os itens e a interpretação do resultado - lesão completa ou incompleta. ${ }^{13}$ Este resultado mostra que os itens da escala traduzida estão corroborando para medir o mesmo construto geral.

De acordo com estudos anteriores, a abordagem de autorrelato é mais eficaz em indivíduos com LME crônica, com pelo menos 1 ano de lesão, sendo menos confiável quando utilizado em lesões mais agudas. ${ }^{4,14} \mathrm{~A}$ análise para a confiabilidade teste-reteste apresentou valores elevados, sinalizando que o indivíduo é capaz de concordar com as respostas extraídas pelas questões propostas, podendo ser considerado um questionário confiável para ser utilizado em indivíduos portadores de LME crônica.

Quando analisada a compatibilidade das respostas entre teste e reteste em cada indivíduo, constatou-se que apenas três indivíduos responderam de forma diferente. Estes indivíduos possuíam classificação AIS C com > 10 anos de lesão. É necessário considerar que a maioria das amostras foi classificada como AIS C (12 indivíduos), dos quais apenas 12,5\% corresponderam à amostra estudada com distinção de resposta. Embora a distribuição da amostra possa influenciar este achado, Hamilton et al., ${ }^{14}$ ao compararem o resultado do questionário do seu estudo com o resultado do exame físico, observaram que o questionário era mais confiável para indivíduos com classificação AIS A e D do que para os indivíduos classificados com AIS B e C. A hipótese levantada pelos autores é que a divergência se deve à maior precisão 
dos resultados quando os pacientes são classificados com AIS A e D, principalmente quando avaliados pelo teste físico. Além disso, os autores enfatizam que o exame físico não deve ser descartado e deve ser realizado sempre que possível e factível, já que oferece uma melhor avaliação destes segmentos. $^{14}$

Embora o questionário não seja $100 \%$ preciso, seu uso pode ser recomendado em determinadas circunstâncias e em determinados pacientes, segundo Harvey et al., ${ }^{4}$ afastandose, assim, a necessidade de submeter os indivíduos com LME ao exame físico, quando isso não for possível ou as condições do local e as técnicas forem consideradas inadequadas. Além disso, em situações como um projeto de pesquisa de base comunitária, onde a avaliação destes segmentos é de pouca relevância, ou mesmo em atendimento ambulatorial que requer avaliações rápidas, o autorrelato pode ser muito útil e capaz de atender às necessidades. ${ }^{4,14}$

\section{Conclusão}

O processo de tradução e adaptação transcultural do questionário (S4-5Q) de autorrelato das funções sensoriais e motoras S4-S5, no que tange a sua aplicação na língua portuguesa brasileira, foi realizado de forma sistemática e exitosa, proporcionando confiabilidade e viabilidade na aplicação deste questionário em indivíduos com LME no Brasil. Sua aplicação é fácil e rápida de realizar durante a avaliação da função sacral motora e sensorial em indivíduos com LME, mas não exclui a realização do exame físico para que se obtenha diagnósticos mais precisos. Ressaltamos que o questionário na versão traduzida para o português teve sua confiabilidade testada apenas com pacientes crônicos (no mínimo 1 ano após a lesão), por ser este o público-alvo sugerido pelo autor da versão original. Além disso, sugere-se que o questionário, quando aplicado após o teste físico dos demais dermátomos, pode proporcionar um melhor entendimento ao indivíduo, já que as perguntas refletem literalmente o teste físico.

Por fim, embora o presente estudo tenha avaliado as equivalências recomendadas para a tradução e adaptação transcultural do questionário S4-5Q de acordo com a metodologia proposta por Beaton et al., ${ }^{11}$ estudos adicionais ainda são necessários para melhor caracterizar a validade conceitual da versão do questionário.

\section{Suporte Financeiro}

Não houve suporte financeiro de fontes públicas, comerciais, ou sem fins lucrativos.

Conflito de Interesses

Os autores declaram não haver conflito de interesses.

\section{Agradecimentos}

Os autores agradecem à professora Lisa Harvey (pesquisadora sênior, Centro John Walsh para Pesquisa em Reabilitação, Sydney, Austrália) por sua assistência na adaptação transcultural do questionário S4-5Q. O presente estudo foi parcialmente financiado pela Coordenação de Aperfeiçoamento de Pessoal de Nível Superior Brasil (CAPES) - Código Financeiro 001.

\section{Referências}

1 Kirshblum S, Botticello A, Benedetto J, et al. A Comparison of Diagnostic Stability of the ASIA Impairment Scale Versus Frankel Classification Systems for Traumatic Spinal Cord Injury. Arch Phys Med Rehabil 2020;101(09):1556-1562

2 Maynard FM Jr, Bracken MB, Creasey G, et al. International standards for neurological and functional classification of spinal cord injury. Spinal Cord 1997;35(05):266-274

3 Kirshblum S, Waring W III. Updates for the international standards for neurological classification of Spinal Cord Injury. Phys Med Rehabil Clin N Am 2014;25(03):505-517vii

4 Harvey LA, Weber G, Heriseanu R, Bowden JL. The diagnostic accuracy of self-report for determining S4-5 sensory and motor function in people with spinal cord injury. Spinal Cord 2012;50 (02):119-122

5 Roberts TT, Leonard GR, Cepela DJ. Classifications In Brief: American Spinal Injury Association (ASIA) Impairment Scale. Clin Orthop Relat Res 2017;475(05):1499-1504

6 Kirshblum S, Botticello A, Lammertse DP, Marino RJ, Chiodo AE, Jha $A$. The impact of sacral sensory sparing in motor complete spinal cord injury. Arch Phys Med Rehabil 2011;92(03):376-383

7 Burns SP, Tansey KE. The Expedited International Standards for Neurological Classification of Spinal Cord Injury (E-ISNCSCI). Spinal Cord 2020;58(06):633-634

8 Zariffa J, Kramer JLK, Jones LAT, Lammertse DP, Curt A, Steeves JD. European Multicenter Study about Spinal Cord Injury Study Group. Sacral sparing in SCI: beyond the S4-S5 and anorectal examination. Spine J 2012;12(05):389-400

9 Marino RJ, Schmidt-Read M, Kirshblum SC, et al. Reliability and Validity of S3 Pressure Sensation as an Alternative to Deep Anal Pressure in Neurologic Classification of Persons With Spinal Cord Injury. Arch Phys Med Rehabil 2016;97(10):1642-1646

10 Liu N, Xing H, Zhou MW, Biering-Sørensen F. Development and validation of a bowel-routine-based self-report questionnaire for sacral sparing after spinal cord injury. Spinal Cord 2017;55(11):1010-1015

11 Beaton DE, Bombardier C, Guillemin F, Ferraz MB. Guidelines for the process of cross-cultural adaptation of self-report measures. Spine (Phila Pa 1976) 2000;25(24):3186-3191

12 Mokkink LB, Terwee CB, Patrick DL, et al. The COSMIN study reached international consensus on taxonomy, terminology, and definitions of measurement properties for health-related patientreported outcomes. J Clin Epidemiol 2010;63(07):737-745

13 Landis JR, Koch GG. The measurement of observer agreement for categorical data. Biometrics 1977;33(01):159-174

14 Hamilton R, Kirshblum S, Sikka S, Callender L, Bennett M, Prajapati P. Sacral examination in spinal cord injury: Is it really needed? J Spinal Cord Med 2018;41(05):556-561 


\section{VERSÃO BRASILEIRA DO QUESTIONÁRIO SENSITIVO E MOTOR DE S4-5 (S4-5Q)}

NOME:

DATA:

AVALIADOR:

\section{Roteiro para administração do questionário sensitivo e motor S4-5 (S45Q):}

Eu vou lhe fazer 4 perguntas sobre a sua sensibilidade e força dentro e ao redor do seu ânus.

As perguntas são de natureza pessoal. Se em algum momento você se sentir constrangido ao responder a qualquer pergunta, ou, por qualquer razão, prefira não respondê-las, me informe por favor, que eu interrompo o questionário. As perguntas são muito importantes para determinar a extensão da sua lesão da medula espinhal. Elas nos informam se as mensagens nervosas estão conseguindo passar pela sua lesão até o final da sua medula espinhal. A outra maneira de se obter a mesma informação é através de um clínico testando a sensibilidade e força de seu ânus. Isso envolve um exame de toque retal. As perguntas nos ajudam a evitar a necessidade de um exame retal, muito embora a decisão final sobre este tipo de exame seja sempre sua. Eventualmente, independentemente de suas respostas às perguntas, nós recomendamos que você se submeta a um exame retal. Caso você não compreenda a terminologia que estamos utilizando, queira por favor responder "não tenho certeza".

Você gostaria de prosseguir e sente-se confortável com as perguntas?

Sim Não

(circule a resposta apropriada)

Pergunta 1a: Você sentiria alguma coisa se eu tocasse levemente com algodão a pele bem ao redor do seu ânus?

Sim Não Não tenho certeza

(circule a resposta apropriada)

Pergunta 1b: Você poderia distinguir entre as extremidades com ou sem ponta de um alfinete de segurança, se eu tocasse a pele ao redor de seu ânus alternadamente com cada uma delas?

Sim Não Não tenho certeza

(circule a resposta apropriada)

Pergunta 1c: Você sentiria pressão se eu inserisse um dedo com luvas no seu reto e aplicasse pressão na parede do seu ânus? Sim Não Não tenho certeza

(circule a resposta apropriada)

Pergunta 2: Você consegue contrair os músculos do seu ânus como se fosse segurar a vontade de evacuar, ou segurar um enema, ou para evitar a saída de gases?

Sim Não Não tenho certeza

(circule a resposta apropriada) 